\title{
PathwAI Systems in Healthcare - a Framework for Coupling AI and Pathway-based Health Information Systems
}

\author{
Tim Scheplitz \\ Technische Universität Dresden \\ tim.scheplitz@tu-dresden.de
}

\author{
Thure Weimann \\ Technische Universität Dresden \\ thure.weimann@tu-dresden.de
}

\author{
Martin Burwitz \\ Technische Universität Dresden \\ martin.burwitz@tu-dresden.de
}

\begin{abstract}
Pathway-based Health Information Systems (HIS) enable planning, execution and improvement of standardized care processes. Adaptive behavior and learning effects are taken to a new level by advances in Artificial Intelligence (AI). Yet, design support to unlock synergies from coupling pathway-based HIS with AI is lacking. This Umbrella Review identifies applied purposes of AI in healthcare, describes the relation to pathway-based HIS, and derives a PathwAI Framework as design support for future research and development activities. Previous findings already provide a large base of approaches to realize personalized care pathways and improve coordination and business operations. Furthermore, potentials for designing learning health systems at micro, meso, and macro levels are formulated, but there is still greater opportunity for future research and design. Pathwaybased HIS in this context can not only provide interpretable and interoperable data input, but can be conceptual as well as operational receivers of artificially generated knowledge.
\end{abstract}

\section{Introduction}

Artificial Intelligence (AI) promises a digital revolution in healthcare and opens up new potentials for transforming the entire care pathway [1]-[3]. Benefits and opportunities of using AI in healthcare particularly rely in decreasing costs and reduction of inefficiencies while improving the care quality with a more personalized, precise and preventive medicine [4], [5]. Instead of replacing human workforce, the partnership of humans and AI may also bring back humanity to medicine and health professionals can spend more time with their patients [6], [7]. For example, AI-driven applications can support radiologists by diagnosing diseases or even replace particular tasks [1], [8]. Recent achievements of AIbased speech recognition allow to communicate with computers as we do with humans and accelerate documentation tasks of health professionals [3] or facilitate the interaction with virtual coaching systems that support patients in their daily life [2].

While the mentioned examples focus on single interventions, AI may be also used to learn about entire care pathways. Especially against the background of chronic disease scenarios (e.g. cancer, diabetes or multiple sclerosis), that often involve a lifelong patient journey and long-term care, an investigation of the whole pathway is of particular interest [9]. Instead of focusing on single interventions, we experienced in several digital health research projects the benefits of process orientation to find the best holistic approach of digital support to those care scenarios. We therefore put care pathways in the center of our innovation activities to design pathway-based Health Information Systems (HIS), i.e., systems that support processcentered care scenarios [10], [11]. Notably the emergence and availability of national Electronic Health Records (EHRs) that capture the whole patient history across institutions could further drive pathwaybased HIS [12]. Analyzing this multimodal data (e.g. diagnoses, conducted treatments, medical parameters or unstructured clinical notes) with advanced analytical techniques of AI could lift pathway-based HIS to a new level [11]. Thus, instead of relying on static process knowledge and manual adjustments based on explicit expert knowledge, coupling AI with pathway-based HIS promises more dynamic and new insights into existing care pathways.

However, to the best of our knowledge, no study has reviewed the approaches and design options of how AI can enrich pathway-based HIS. Therefore, we want to investigate the following research questions:

RQ1: How have pathway-based HIS been enriched by AI so far?

RQ2: What needs a framework that might assist the design and implementation of AI application in relation to pathway-based HIS? 
This paper is structured as follows. In the next section, we introduce the theoretical background on pathway-based HIS and AI in healthcare and motivate the general analysis concept of our work. Section 3 describes the research methodology followed by our results in section 4 (RQ1) and 5 (RQ2). The paper closes with a discussion and an outlook on future research opportunities.

\section{Background}

\subsection{Care pathways and associated systems}

A Care Pathway (CP) is a specific, standardized description of a clinical process for a defined combination of symptoms adapted to clinical conditions [13], [14]. It is a multidisciplinary tool to improve quality of care for a specific patient type and to achieve a higher degree of efficiency and a higher grounding in the evidence base [15]. Accordingly, CPs are used as communication tools between professional caregivers to manage and standardize care [16]. Different terms are used to emphasize specific peculiarities and scopes of CPs, such as intraorganizational (clinical pathways), inter-institutional (integrated pathways) or patient-centered (patient pathways) orientations [15], [17], [18]. The common ground is to provide an integrating process view on the care-relevant items, including medical, organizational and administrative aspects, to support the planning, execution and improvement of medical care.

Depending on the maturity of Information and Communication Technology (ICT), the previous usage of CPs seldomly went beyond the organizational level (e.g. checklists, local pdf-files, printed management manuals). But meanwhile, ICT landscapes are starting to change and to adapt process knowledge into HIS design. The resulting continuum of HIS's process awareness goes from not aware to process-aligned HIS design (process-centered HIS) until real pathway-based HIS, where the $\mathrm{CP}$ is a configuring part of the running system. Thus, there are several HIS implementations for a workflow-oriented support of daily care processes [19]-[21], and systems that support process-centered care scenarios [10], [11].

Considering the planning of medical care, HIS can support the design of CPs as well as their adaptation for a specific patient, i.e., mapping patient conditions to $\mathrm{CP}$ scopes to identify and customize the best matching $\mathrm{CP}$ for an individual case. During the following stage of CP execution, HIS can provide guidance along the CP-defined care process concerning communication and documentation but also support monitoring and adaptation of the specific care process to topical patient conditions. The collected data during $\mathrm{CP}$ execution can provide a profound knowledge base for process evaluation and improvement. Based on the data, HIS will be able to support a continuous evaluation and improvement, both, of specific patient-individual CP instances (adhoc) and of the underlying CP itself.

\subsection{Rising AI in healthcare}

Driven by advances in computing power, machine learning and its subfield deep learning has been on the rise since the beginning of the 2000's [22]. Machine Learning (ML) can be considered as automated analytical model building for conducting cognitive tasks and addresses the drawbacks of handcrafted rules [23]. Therefore, ML is nowadays in the center of AI research in healthcare [24]. A term related to ML is "data mining" and can be understood as the process of building ML models [25]. With respect to business processes, the field of process mining has emerged in the last decades and investigates ML approaches to gain knowledge by analyzing event logs [26].

In general, ML techniques can be classified along the three types supervised learning, unsupervised learning and reinforcement learning [23]. Supervised learning refers to problems where a target variable should be predicted and a training dataset with input data (x) and output data (y) is available [23]. Supervised learning is suited to solve classification problems, i.e. prediction of a categorical variable (e.g. disease diagnosis based on a CT image as input), or regression problems, i.e., predicting a continuous variable (e.g. prediction of blood sugar level) [27]. Typical supervised learning techniques are linear and logistic regression, decision trees or Artificial Neural Networks (ANNs) [27]. The idea of deep learning builds on the latter technique (ANNs) and uses complex architectures with deeply stacked layers to increase learning capabilities [23]. In contrast, unsupervised learning aims to generate knowledge about data (patterns and correlations) without a target variable (y) being specified [27]. One typical unsupervised learning technique is clustering (e.g. dividing data from multiple patients into groups of similar patients) [27]. Unlike supervised and unsupervised learning, reinforcement learning doesn't need a training data set beforehand [23]. Algorithms from the field of reinforcement learning (e.g. Qlearning) solve sequential decision making problems to achieve a certain goal by building a model in a "trial and error" process or by expert demonstrations [28]. Hence, a software agent interacts with an environment by taking certain actions that change the environment's state and receives rewards in response to the actions [27]. For healthcare, reinforcement 


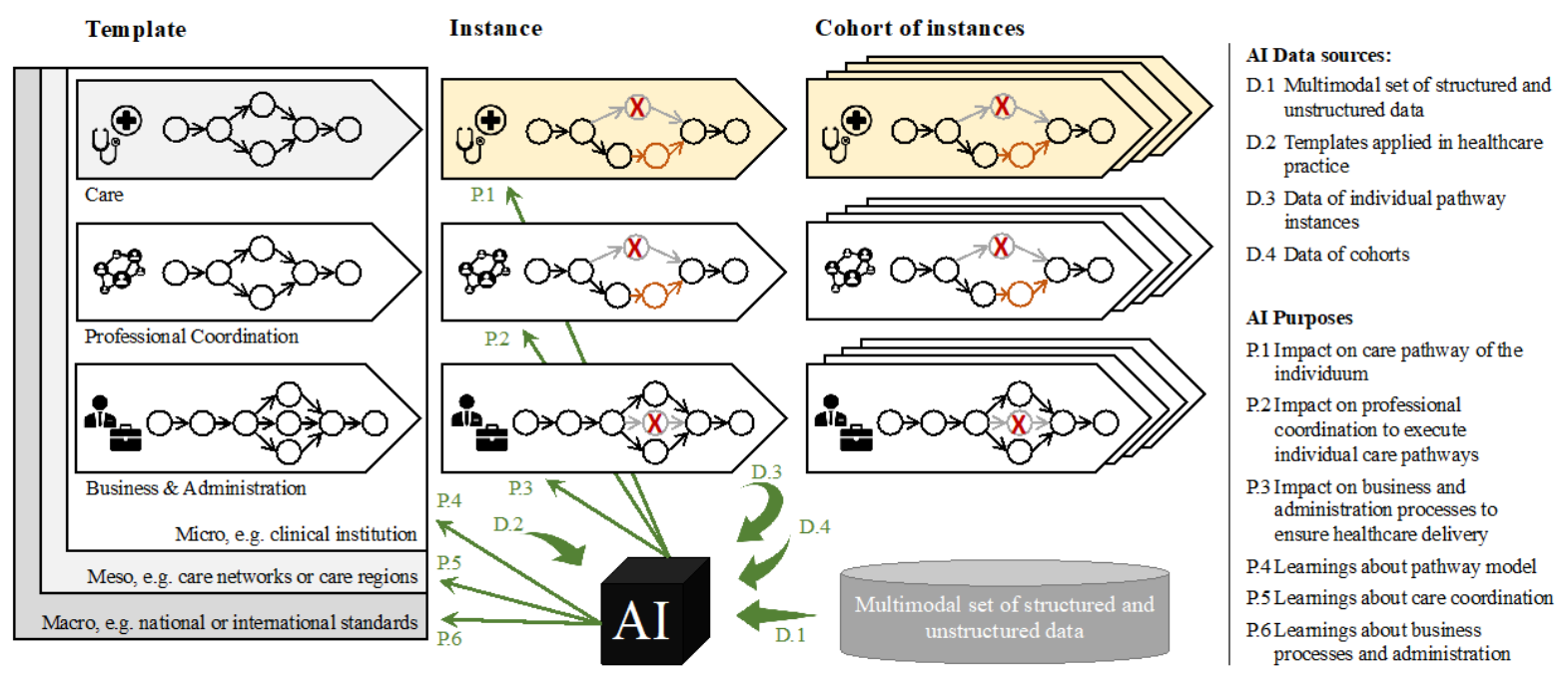

Figure 1. Analysis concept of coupling Al and pathway systems

learning enables personalized treatments such as optimizing therapy plans to the patient and the clinical goals [29], [30].

\subsection{Conceptualization of investigation}

Considering the variety of mechanisms how AI is able to generate effects in healthcare, there are multiple possibilities to enrich pathway-based HIS with such techniques. We developed an analysis concept that describes general alternatives of AI integration in the context of pathway-based HIS (see Figure 1). It comprises the following elements:

Pathway Template: Model of a care process; Represents standardized process knowledge; Differentiation by views on medical care, coordination of professional care delivery and business as well as administration; Different reference levels describe intra-organizational (micro), cross-institutional (meso), and national or international (macro) levels.

Pathway Instance: Application of the pathway model for a patient; process execution on all views (care, professional coordination, business \& administration); pathway systems as application systems to apply, execute and store pathway instances.

Cohorts of pathway instances: Selected set of pathway instances; Cohort building by e.g. indication, symptom, treatment, demographics, time period, etc.

Data sources: Multimodal set of structured and unstructured data; variety of HIS, application systems and devices including, e.g. EHR-systems, clinical information systems, health information data bases, med-tech devices, patient's IT in home environment.

$A I$ : interpreted as black box of algorithms and techniques to generate knowledge from different data in an automated way for defined purposes.
With the simplified understanding of AI as a black box, two aspects come into focus of the investigation. First, for which purposes is AI attempted. And second, what data is needed as input for these purposes. We assume that AI might be coupled with pathway-based HIS to fulfill one or more of these six top-level purposes (see Figure 1). These top-level purposes are analyzed more in detail by this study. Thus, we examine how AI supports ongoing care (P1-P3) as well as general learning effects at micro, meso or macro level (P4-P6). We further assume that AI could basically use four types of data input: multimodal set of structured and unstructured data (D.1), templates applied in healthcare practice (D.2), data of individual pathway instances (D.3) or data of multiple pathway instances selected based on a certain criterion, i.e., cohort of instances (D.4). This proposed distinction is primarily qualitative in nature and should not be understood as physically delineable data repositories. Rather, data structures of pathway-based HIS should be delineated from the set of other data sources. Such data structures represent temporally and causally related sequences of activities as well as conditions and reference applied pathway models, treatment plans, or care cases.

\section{Methodology}

The recent popularity of AI in healthcare promoted primary studies as well as reviews. In order to align the knowledge aggregated therein with our own objective, an umbrella review was conducted to analyze a large amount of literature in a targeted manner [31]-[33]. Its analytical results were combined with our analysis concept to derive the PathwAI 
Framework that seeks to support the design of intelligent, pathway-based HIS.

The literature selection was conducted in end of June 2021. Three databases have been selected for identification of relevant IS or medical literature: IEEE Xplore, AIS Library and PubMed. We therefore used the search strategy mentioned in Table 1. We decided to focus on a short and current time range to balance up-to-dateness of a review of reviews with its retrospectivity.

Table 1. Search strategy of umbrella review

\begin{tabular}{ll} 
Data field & Terms \\
\hline Title & "artificial intelligence" OR "AI" OR "ML" OR \\
& "learning" OR "mining" \\
Title & "health*” OR "medic*" OR "care" \\
Abstract & "review" \\
Year & 2018-2021
\end{tabular}

Details of selection process are given in Figure 2. We only include articles that a) do not focus on a single AI technique; b) do not focus only one specific medical field, disease, symptom, treatment or intervention; c) state details of review strategy and d) discuss findings in relation to CPs and/or associated processes. Considering the continuum, from not aware to process-centered HIS until pathway-based HIS (see section 2.1), we rather remained open minded for different technological solutions than stuck to a strict inclusion criterion on dedicated types of systems. Both such a definition and its application would have been difficult to implement. Finally, seven articles matched the inclusion criteria for full text analysis (Table 2).

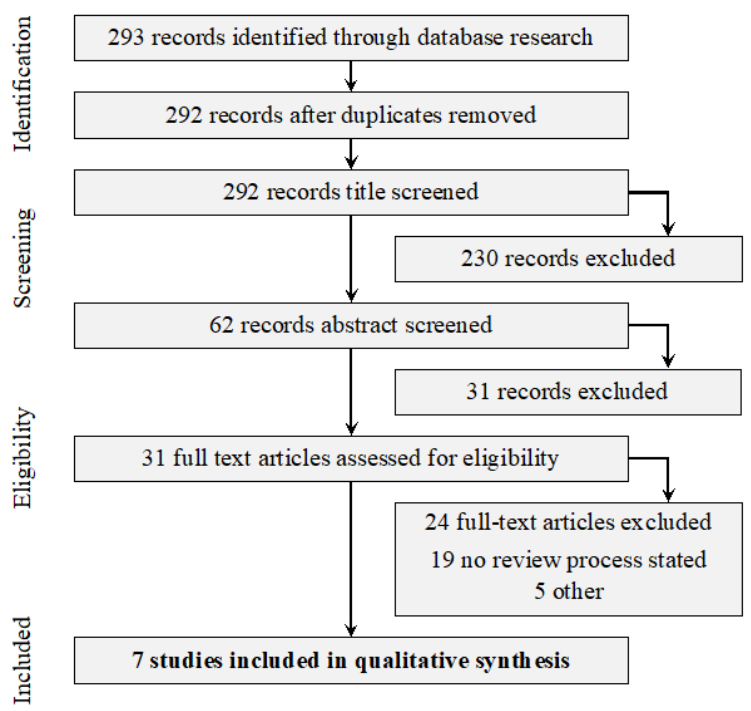

Figure 2. Review details according to PRISMA [34]
Table 2. Analyzed reviews sorted by year

\begin{tabular}{|c|c|c|}
\hline Authors & Title & Year \\
\hline $\begin{array}{l}\text { Kueper et al. } \\
\text { [35] }\end{array}$ & $\begin{array}{l}\text { Artificial Intelligence and Primary Care } \\
\text { Research: A Scoping Review }\end{array}$ & 2020 \\
\hline $\begin{array}{l}\text { Triantafyllidis } \\
\& \text { Tsanas [36] }\end{array}$ & $\begin{array}{l}\text { Applications of Machine Learning in } \\
\text { Real-Life Digital Health Interventions: } \\
\text { Review of the Literature }\end{array}$ & 2019 \\
\hline $\begin{array}{l}\text { Shickel et al. } \\
\text { [37] }\end{array}$ & $\begin{array}{l}\text { Deep EHR: A Survey of Recent } \\
\text { Advances in Deep Learning Techniques } \\
\text { for EHR Analysis }\end{array}$ & 2018 \\
\hline $\begin{array}{l}\text { Erdogan \& } \\
\text { Tarhan [38] }\end{array}$ & $\begin{array}{l}\text { Systematic Mapping of Process Mining } \\
\text { Studies in Healthcare }\end{array}$ & 2018 \\
\hline $\begin{array}{l}\text { Batista \& } \\
\text { Solanas [39] }\end{array}$ & $\begin{array}{l}\text { Process Mining in Healthcare: A } \\
\text { Systematic Review }\end{array}$ & 2018 \\
\hline Xiao et al. [40] & $\begin{array}{l}\text { Opportunities and challenges in } \\
\text { developing deep learning models using } \\
\text { electronic health records data: a } \\
\text { systematic review }\end{array}$ & 2018 \\
\hline $\begin{array}{l}\text { Islam et al. } \\
\text { [41] }\end{array}$ & $\begin{array}{l}\text { A Systematic Review on Healthcare } \\
\text { Analytics: Application and Theoretical } \\
\text { Perspective of Data Mining }\end{array}$ & 2018 \\
\hline
\end{tabular}

\section{Findings}

\subsection{Purposes of $\mathrm{AI}$ mentioned in reviews and implementation approaches}

As summarized in Table 3, there are several AI techniques conceivable to address the defined purposes. Therefore, we want to focus on a subset of techniques that were particularly emphasized in the reviewed articles and concisely state how they could leverage pathway-based HIS. One promising AI technique for analyzing $\mathrm{CPs}$ represented as the longitudinal sequential patient history in EHRs are concept embeddings [37], [40]. Against the background that CPs can span several decades of lifetime, the idea behind medical concept embeddings is to generate aggregated representations of medical concepts in the pathway. These aggregations are lowdimensional vectors capturing the latent relationships (e.g. preceding and succeeding diagnoses or treatments) [37]. Therefore, approaches originating from the field of Natural Language Processing (NLP) are used such as word2vec (capturing the context of words in a corpus) with adaptations for the medical domain (e.g. med2vec) [37], [42]. For instance, the skip-gram architecture of ANNs [40] can be used to predict the context for a certain input word or in case of CPs, predict adjacent diagnoses or treatments based on a certain medical concept. As an example, one could imagine that a predecessor of a CT scan of the abdomen could be abdominal pain, followed by a surgery to remove the appendix after imaging. However, common approaches based on word2vec generated concept embeddings fail to capture the 
actual timestamp relationships, i.e., it is not represented if event $B$ happened one week or one year before event A (see Zhu et al. [43] for an advanced approach). While a medical concept embedding thus may provide new insights into a cohort of pathway instances, one can go one step further and derive also abstract patient representations. This can be achieved by aggregating the medical concept embeddings contained in the CP of the patient [44]. In a next step, similarity metrics can be calculated between pairwise patient representations and may facilitate scenarios such as analysis of comparative treatment effectiveness and personalized medicine [43]. Furthermore, clinical phenotyping can be considered as an application of concept embeddings by matching patient vectors to a defined phenotype vector [40]. In addition, one can use the abstract patient representations as input for predictive models (e.g. to predict risks or clinical outcomes). Several studies also reveal that this approach can increase the predictive performance compared to raw data input [37].

Considering that pathway data is sequential in nature, Recurrent Neural Networks (RNNs), a special type of ANNs that are able to capture long-term dependencies, are particularly suitable for prediction problems [37], [40]. In general, for supporting diagnostics and therapy, one may distinguish with respect to prediction tasks between identification and classification of diseases, as well as sequential prediction of diagnoses and clinical events. With respect to the output, one may further distinguish between outputs without (e.g. general risk for a certain disease) and with temporal constraints (e.g. timespan to next hospital visit and diagnosis) [37]. Furthermore, identified purposes that also rely on prediction problems encompass referral support, forecasting of service demand, disease or infection control, patient management and efficiency improvement.

In addition to these techniques which are particularly rooted in the field of deep learning, there is a vast body of literature on techniques from the process mining field. For example, clustering is often considered as a pre-processing step in the discovery of new processes, i.e., aggregating the event logs of a cohort of pathway instances to identify the underlying pathway template(s) [38], [39]. Frequently used clustering algorithms with respect to process mining are Trace Clustering, K-Means and Hierarchical clustering algorithms, whereas for process discovery, the Heuristic Miner and Fuzzy Miner algorithms dominate in the literature [38], [39]. Based on the discovered processes, templates may be derived that could serve to check on how a business process or $\mathrm{CP}$ instance align with the template (conformance checking) [39]. Furthermore, there are process mining techniques available to extend the pathway template based on the observed pathway instances (process enhancement) [39].

Last but not least, given that structured event logs with timestamps are often not available, as EHRs usually contain mixed data (structured and unstructured), advanced information extraction techniques may be applied to reconstruct and derive the CP. Especially for dealing with unstructured data (e.g. clinical notes), advanced NLP techniques are particularly useful for extracting single concepts, temporal events, relations or expand abbreviations [37], [40], [41].

\subsection{Allocation to top-level purposes}

Within the interpretation phase, we were able to make an argumentative allocation to the defined toplevel purposes of our analysis concept. Rarely was a one-to-one mapping identified (e.g. Disease identification and classification for P.1). More frequently, assignments to two to three top-level purposes were determined. The close connection between the process views (medical, coordination, business), a primarily abstract description of the purposes by the reviews, and the logical coexistence of added benefits are the main reasons for this. The latter can be illustrated by clinical phenotyping. On the one hand, knowledge about phenotypes is extracted as a model (P.4), and on the other hand, patients (pathway instances) can subsequently be assigned to learned phenotypes for individualization of care processes (P.1). The allocation made (see Table 3 and Figure 3) leaves room for further interpretation and should therefore be classified as a suggestion by the authors.

\subsection{Relation to pathway-based HIS}

In addition to conceptual links, systemic input and output relationships were also explored to describe the role of pathway-based HIS in interaction with AI. Even authors of analyzed reviews did not mention explicitly the relation between AI applications and pathway concept or realizing pathway-based HIS, their contributions indicate implicitly whether pathway-based HIS do "only" provide interpretable and interoperable data as input of AI techniques or pathway based HIS and AI occur in a synergetic loop, where outputs of AI affect pathway models or instances. These analysis results are embedded within the PathwAI Framework (see Figure 3) and also leaves room for further interpretation. Again, it should therefore be classified as a suggestion by the authors and used as aid for architectural design activities. 
Table 3. Findings of umbrella review - purposes of Al application in relation to pathway-based HIS

\begin{tabular}{|c|c|c|c|}
\hline $\begin{array}{l}\text { Purpose of AI } \\
\text { application }\end{array}$ & Description & $\begin{array}{l}\text { Purpose } \\
\text { group }\end{array}$ & Ref \\
\hline $\begin{array}{l}\text { Diagnostic and } \\
\text { therapeutic decision } \\
\text { support }\end{array}$ & $\begin{array}{l}\text { AI provided information to inform diagnosis and treatment decisions. Diagnostic } \\
\text { applications typically seek for onset or probability that a patient has a particular } \\
\text { condition or recommend diagnosis categories. Diagnostic decision support is used to } \\
\text { describe and/or predict various conditions or events. Therapeutic decision support } \\
\text { includes any management or care provided (or absence of unnecessary actions) to a } \\
\text { patient with specific health condition(s) or symptom(s). Therapeutic applications } \\
\text { result typically from diagnostic decision support. They are often used to predict or } \\
\text { define a personalized treatment, e.g. medication or treatment plans, for improvements } \\
\text { in quality outcomes or efficiency. }\end{array}$ & P.1, P.2, P.3 & $\begin{array}{l}{[35],[36],} \\
{[40],[41]}\end{array}$ \\
\hline $\begin{array}{l}\text { Disease } \\
\text { identification and } \\
\text { classification }\end{array}$ & $\begin{array}{l}\text { AI used to screen and detect whether specific diseases can be confirmed. Different } \\
\text { types of Classifications are applicable: disease specific (categorical or multi-label) or } \\
\text { disease non-specific (normal, preictal or seizure subject). }\end{array}$ & P.1 & [40], [41] \\
\hline$L \begin{array}{l}\text { Medical concept } \\
\text { embedding }\end{array}$ & $\begin{array}{l}\text { AI used to derive abstract representation of clinical concepts based on analysis of real } \\
\text { cohorts. It aggregates medical concepts that occur frequently together. Concept } \\
\text { embedding is often an intermediate, descriptive step for building a predictive model } \\
\text { of previous and next steps from a certain position in the pathway for better } \\
\text { performance. }\end{array}$ & P.1, P.4 & [37], [40] \\
\hline$\left\llcorner\quad \begin{array}{l}\text { Clinical } \\
\text { phenotyping }\end{array}\right.$ & $\begin{array}{l}\text { AI used to discover phenotypes via feature representation and investigates association } \\
\text { of pathway instance to different phenotypes. First, phenotypes are extracted as new } \\
\text { knowledge out of cohorts of instances, e.g. by prevalence of a condition or patterns of } \\
\text { patient profiles. Second, single instances are matched with discovered phenotypes. } \\
\text { Third, treatments might be personalized, e.g. by pathway instance adaptions. Clinical } \\
\text { phenotyping is considered as a type of concept embedding. }\end{array}$ & P.1, P.4 & $\begin{array}{l}{[35]-[37],} \\
{[40]}\end{array}$ \\
\hline$\downarrow \begin{array}{l}\text { Sequential } \\
\text { prediction in } \\
\text { diagnostics }\end{array}$ & $\begin{array}{l}\text { AI predicts future diagnoses based on past longitudinal event sequences (patient's } \\
\text { history), e.g. onset of new disease condition, risk of in-hospital mortality, discharge } \\
\text { diagnoses. Differentiation of static (categorical or numeric) or temporal (time stamp } \\
\text { or range included in prediction) }\end{array}$ & P.1 & [37], [40] \\
\hline$\downarrow \begin{array}{l}\text { Sequential } \\
\text { prediction of } \\
\text { clinical events }\end{array}$ & $\begin{array}{l}\text { AI predicts future clinical events based on past longitudinal event sequences } \\
\text { (patient's history), e.g. unplanned hospital admission/ readmission, length of stay. } \\
\text { Differentiation of static (categorical or numeric) or temporal (time stamp or range } \\
\text { included in prediction) }\end{array}$ & P.2, P.3 & $\begin{array}{l}{[36],[37],} \\
\quad[40]\end{array}$ \\
\hline Process clustering & $\begin{array}{l}\text { AI identifies groups of similar business processes or care pathways based on analysis } \\
\text { of a cohort of instances. }\end{array}$ & P.1, P.2, P.3 & [38], [39] \\
\hline $\begin{array}{l}\text { Process discovery and } \\
\text { proof of conformance }\end{array}$ & $\begin{array}{l}\text { AI used to derive retrospectively or ad hoc business process or care pathway instance } \\
\text { based on analysis of a single or a cohort of instances. Often follows a check on how a } \\
\text { business process or care pathway instance align with the underlying template. }\end{array}$ & P.1, P.2, P.3 & [38], [39] \\
\hline Referral support & $\begin{array}{l}\text { AI provided information to support decisions about referring patients to specialist } \\
\text { services or AI assisted with technical aspects of the referral process. }\end{array}$ & P. 2 & {$[35]$} \\
\hline $\begin{array}{l}\text { Health care utilization } \\
\text { analyses }\end{array}$ & $\begin{array}{l}\text { AI provided information about interactions with or processes within health care } \\
\text { systems, for example frequency or quantity of patient visits. }\end{array}$ & P.2, P.3 & {$[35]$} \\
\hline $\begin{array}{l}\text { Forecasting of service } \\
\text { demand }\end{array}$ & AI used to predict demand of healthcare services on macro level & P.2, P.3 & [41] \\
\hline $\begin{array}{l}\text { Disease or infection } \\
\text { control }\end{array}$ & AI used to monitor and predict dynamic of diseases or infections on macro level & P.2, P.3 & [41] \\
\hline Efficiency improvement & $\begin{array}{l}\text { AI used to predict service demand on micro level and improve resource utilization } \\
\text { and allocation (decision support) }\end{array}$ & P.2, P.3 & {$[41]$} \\
\hline Patient management & $\begin{array}{l}\text { AI used to adapt scheduling and forecasting based on patient conditions and behavior } \\
\text { (decision support) }\end{array}$ & P.2, P.3 & [41] \\
\hline $\begin{array}{l}\text { Performance } \\
\text { quantification }\end{array}$ & AI used to quantify performance of medical service delivery & P.3 & [41] \\
\hline Information extraction & AI used to extract knowledge from structured or unstructured data & P.4, P.5, P.6 & [35], [41] \\
\hline$L \begin{array}{l}\text { Process } \\
\text { enhancement }\end{array}$ & AI used to extent pathway templates with information from event logs. & P.4, P.5, P.6 & [38], [39] \\
\hline $\begin{array}{l}\text { Derivation of } \\
\text { healthcare } \\
\text { programs }\end{array}$ & AI used to improve design of national healthcare programs (macro level) & P.4, P.5, P.6 & [41] \\
\hline
\end{tabular}




\section{PathwAI Framework}

The PathwAI Framework (see Figure 3) sorts the identified purposes of AI applications to enhance toplevel impacts on individual CPs (P.1), its coordination (P.2) and related business as well as administration processes (P.3). Impacts on these intertwined process views effect ongoing individual care delivery and may result consequently in improved care delivery of population. Besides this distinction, the proposed framework further indicates purposes by its context of learning: learnings about pathway models (P.4), learnings about care coordination (P.5) and learnings about business processes and administration of healthcare (P.6). Here, AI is applied to extract knowledge that should be used to develop new pathway models (templates) on micro (e.g. institutional procedures), meso (e.g. integrated pathway of care networks) and macro level (e.g. national guidelines).

Either new AI applications tend to one or more of the purposes mentioned, all techniques require a sound data input that ensure interpretability and interoperability. Pathway-based HIS could therefore play a critical role, take the potentials to a new level and master the challenge of data accessibility and quality. Such systems are able to ensure comprehensive and structured data sets of individual pathway instances (D.3) that are linked to selected templates (D.2) and in consequence of cohorts of specific interest (D.4). Paired with the increasing amount of accessible and valid health information from various data sources like EHR, smart devices of patients as well as specialized medical databases (D.1), the coupling of AI and pathway-based HIS promises impactful and strong support for individualized care and learning health systems.

Data input

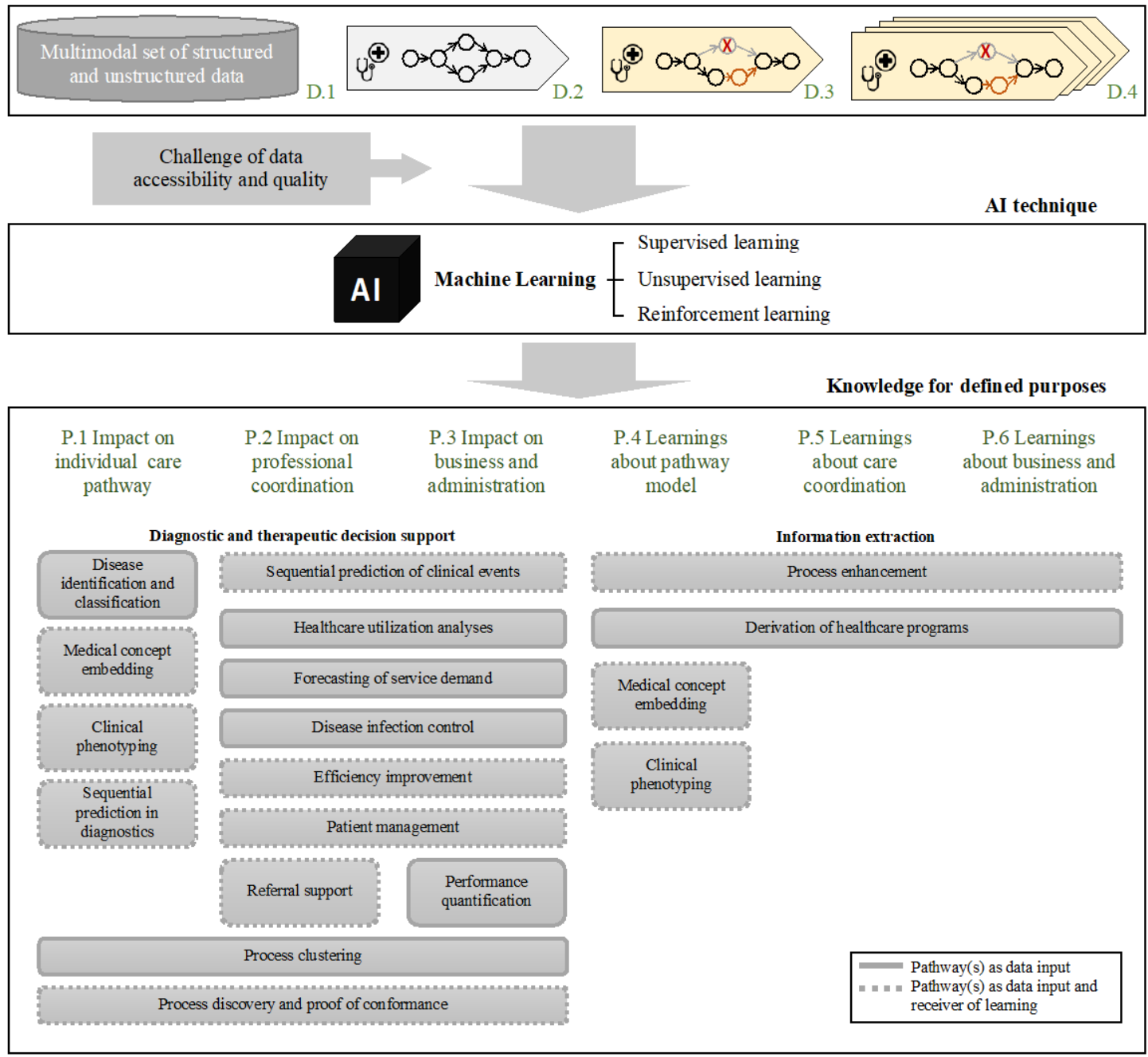

Figure 3. PathwAI Framework - pathway-oriented application of Al in healthcare 


\section{Discussion}

\subsection{Limitations}

As a qualitative research paper, the presented contributions are subject to some limitations. While the selection and analysis of the literature was conducted independently by two researchers, limitations of objectivity remain due to the authors interpretations. Additionally, umbrella reviews seek to interpret prior interpretations done by the authors of selected reviews which are also limited in its objectivity. This "inheritance of subjectivity" might be counteracted by the inclusion criterion of required review strategies. Looking at the parameters of the selection process and the list of analyzed contributions, two further limiting aspects can be identified: Comprehensiveness and timeliness.

6.1.1 Comprehensiveness. In the final proof of eligibility, 18 of 31 articles were excluded due to missing or insufficient description of review details. It is not always clear whether this is reasoned due to a lack of a systematic procedure or an unsatisfactory presentation. Either way, we decided to look back on excluded papers of this last selection step. Thus, we checked three additional articles with the most intensified resonance [1], [45], [46] (count of citations assessed via Google Scholar).

6.1.2 Timeliness. The high number of primary studies reflects the topic's current relevance. However, the speed of technological progress contrasts the fact that five out of eight articles were published in 2018. Thus, the critique occurs that recent progress could not be sufficiently taken into account. Also, reviews, especially umbrella reviews, hold a retrospective analysis position and generate only limited insights for the present and future. Analogous to the previous critique, we additionally checked three articles published in 2021 to strengthen the awareness of current research [47]-[49].

\subsection{Consideration of additional reviews}

6.2.1 Reviews of high relevance. The requirement of a presented systematic review process excluded three articles which enjoy increased attention in the scientific discourse. Since IS research should benefit from review methods of other disciplines [50], these articles were selected to identify additions to previous findings. All in all, the additional reviews align with our assumptions stated in section 2.3 and generally confirm our framework. But they highlight those top- level purposes, that were not discussed prominently within the analyzed reviews (P.4, P.5, P.6).

In their review paper in Nature Medicine journal, He et al. show further alternatives, including: treatment alignment with guidelines (P.1), efficiency increase of hospital management (P.5, P.6), epidemiological registries for population management (P.5, P.6), outcome assessment on quality (P.5, P.6) and the development of disease prevention guidelines (P.5, P.6) [1].

Ching et al. describe AI applications in diagnostics, biological studies and therapeutics, especially in medications [45]. They point out, that AI is also applied for longitudinal analysis for high value knowledge extraction in different contexts (P.4, P.5, P.6). They differentiate the potential to improve choices of interventions (P.1) and methods for the development of new interventions (P.4). Thereby, the prediction of actions to change the outcome in a certain way are even more challenging than prediction of outcomes under specified conditions.

Miotto et al. summarize in their review, how AI is able to use EHR data for prediction and classification tasks based on a patient's history [46]. Comparing the findings of this umbrella review, their results primarily confirm purposes of AI applications that enhance quality of diagnostic or therapeutic interventions.

6.2.2 Recently published reviews. Also, recent publications confirm the PathwAI Framework. The review of Enticott et al. focusses rather on data driven scenarios in Learning Health Systems than on applied AI techniques, which principally correspond to toplevel purposes on knowledge extraction (P.4, P.5, P.6) [48]. The authors highlight benefits in evidence-based care (P.4), clinical organization or system-level performance (P.5, P.6) and concretize it, e.g. compliance with clinical guidelines and a coexistence of standardization and specialization of care.

Koteluk et al. and Bharadwaj et al. confirm benefits in diagnostic and therapeutic processes especially in terms of personalization, organization and velocity of action [47], [49].

\subsection{Future research}

The results of our review reveal that processrelated applications of $\mathrm{AI}$ are well established so far and under ongoing research for realizing personalized and efficient CPs including their organization, coordination and management. Further, the results indicate that synergies can be unlocked by design and implementation of intelligent, pathway-based HIS. Hence, a design method or design principles to support those activities shall be investigated in subsequent 
work. However, the results also show that research on applications for gaining general knowledge about pathway templates are still in its infants but deep learning approaches could make a significant contribution here in the future. Likewise, the application of AI techniques to learn especially on meso and macro layer has so far been little described in the literature and could be addressed in future research. With regard to achieving the notion of personalized medicine, AI-empowered clinical phenotyping will be a significant driver. Especially with advanced and ubiquitous digital health applications on the patients' side such as "virtual coaches", new data can be obtained that could enable new insights on CPs outside the clinical environment. Additionally, future research should elaborate how reinforcement learning could leverage pathwaysupporting HIS. Reinforcement learning could provide a powerful framework for fully autonomous and adaptive CPs (similar to autonomous driving) but patient safety considerations should always come first [51]. Finally, future studies should demonstrate the usefulness of the proposed framework as guidance for designing and implementing AI applications with relation to pathway-based HIS.

\section{Conclusion}

This umbrella review offers a high-level overview on how HIS can be improved by ensuring synergies of pathway-based HIS and the application of AI within such HIS landscapes. A systematized analysis of purposes and interdependencies led to the proposed PathwAI Framework. It aims to guide research and development teams in designing data-driven, learning HIS. It offers a structured view on the bandwidth of possible improvements in healthcare and, thus, guidance for interdisciplinary innovation teams of clinicians, technologists and health systems managers. This study finally provides evidence on AI applications that have already been investigated, developed and applied in relation to CPs. Future research shall further focus on the symbiosis of AI within pathway-based HIS to ensure adaptive, multilevel learning and high-performance HIS.

\section{References}

[1] J. He, S. L. Baxter, J. Xu, J. Xu, X. Zhou, and K. Zhang, "The practical implementation of artificial intelligence technologies in medicine," Nat. Med., vol. 25, no. 1, pp. 30-36, 2019.

[2] E. J. Topol, "High-performance medicine: the convergence of human and artificial intelligence," Nat. Med., vol. 25, no. 1, pp. 44-56, Jan. 2019.
[3] T. Davenport and R. Kalakota, "The potential for artificial intelligence in healthcare," Future Healthc. J., vol. 6, no. 2, pp. 94-98, Jun. 2019.

[4] E. Racine, W. Boehlen, and M. Sample, "Healthcare uses of artificial intelligence: Challenges and opportunities for growth," Healthc. Manage. Forum, vol. 32, no. 5, pp. 272-275, Sep. 2019.

[5] K. B. Johnson et al., "Precision Medicine, AI, and the Future of Personalized Health Care," Clin. Transl. Sci., vol. 14, no. 1, pp. 86-93, Jan. 2021.

[6] L. A. Celi, B. Fine, and D. J. Stone, "An awakening in medicine: the partnership of humanity and intelligent machines," Lancet Digit. Health, vol. 1, no. 6, 2019.

[7] A. L. Fogel and J. C. Kvedar, "Artificial intelligence powers digital medicine," Npj Digit. Med., vol. 1, no. 1, p. 5, Dec. 2018, doi: 10.1038/s41746-017-0012-2.

[8] H. Tizhoosh and L. Pantanowitz, "Artificial intelligence and digital pathology: Challenges and opportunities," J. Pathol. Inform., vol. 9, no. 1, p. 38, 2018.

[9] P. Richter and H. Schlieter, "Paving the Way for Patient Pathways: Synthesizing a User-Centered Method Design with Results from a Systematic Literature Review," 2020.

[10] M. Benedict, H. Schlieter, M. Burwitz, T. Scheplitz, M. Susky, and P. Richter, "A Reference Architecture Approach for Pathway-Based Patient Integration," in 2019 IEEE 23rd International Enterprise Distributed Object Computing Conference, Paris, France, 2019.

[11] H. Schlieter, M. Benedict, K. Gand, and M. Burwitz, "Towards Adaptive Pathways: Reference Architecture for Personalized Dynamic Pathways," in 2017 IEEE 19th Conference on Business Informatics (CBI), Thessaloniki, Greece, Jul. 2017, pp. 359-368.

[12] L. Stegemann and M. Gersch, "The Emergence and Dynamics of Electronic Health Records-A Longitudinal Case Analysis of Multi-Sided Platforms from an Interoperability Perspective," in Proceedings of the 54th Hawaii International Conference on System Sciences, 2021, p. 6183.

[13] L. De Bleser, R. Depreitere, K. De Waele, K. Vanhaecht, J. Vlayen, and W. Sermeus, "Defining pathways," J. Nurs. Manag., vol. 14, no. 7, 2006.

[14] G. Schrijvers, A. van Hoorn, and N. Huiskes, "The care pathway: concepts and theories: an introduction," Int. J. Integr. Care, vol. 12, no. Special Edition Integrated Care Pathways, 2012.

[15] L. Kinsman, T. Rotter, E. James, P. Snow, and J. Willis, "What is a clinical pathway? Development of a definition to inform the debate," BMC Med., vol. 8, pp. 31-33, 2010.

[16] K. Vanhaecht et al., "Prevalence and use of clinical pathways in 23 countries - an international survey by the European Pathway Association," J. Integr. Care Pathw., vol. 10, no. 1, pp. 28-34, Apr. 2006.

[17] P. Richter and H. Schlieter, "Understanding Patient Pathways in the Context of Integrated Health Care Services-Implications from a Scoping Review," presented at the Wirtschaftsinformatik 2019, Siegen, Feb. 2019.

[18] K. Vanhaecht, M. Panella, R. van Zelm, and W. Sermeus, "An overview on the history and concept of 
care pathways as complex interventions," Int. J. Care Pathw., vol. 14, no. 3, pp. 117-123, Sep. 2010.

[19] M. Reichert and P. Dadam, "ADEPT flex — supporting dynamic changes of workflows without losing control," J. Intell. Inf. Syst., vol. 10, no. 2, pp. 93-129, 1998.

[20] Z. Huang, X. Lu, and H. Duan, "Using Recommendation to Support Adaptive Clinical Pathways," J. Med. Syst., vol. 36, no. 3, 2012.

[21] M. Burwitz, H. Schlieter, and W. Esswein, "Agility in medical treatment processes - a model-based approach," in Modellierung 2012, Bonn, 2012.

[22] V. Kaul, S. Enslin, and S. A. Gross, "History of artificial intelligence in medicine," Gastrointest. Endosc., vol. 92, no. 4, pp. 807-812, Oct. 2020.

[23] C. Janiesch, P. Zschech, and K. Heinrich, "Machine learning and deep learning," Electron. Mark., 2021.

[24] R. Challen, J. Denny, M. Pitt, L. Gompels, T. Edwards, and $\mathrm{K}$. Tsaneva-Atanasova, "Artificial intelligence, bias and clinical safety," BMJ Qual. Saf., vol. 28, no. 3, pp. 231-237, 2019.

[25] N. Kühl, M. Goutier, R. Hirt, and G. Satzger, "Machine Learning in Artificial Intelligence: Towards a Common Understanding," 2019.

[26] W. van der Aalst, Process Mining. Berlin, Heidelberg: Springer Berlin Heidelberg, 2016.

[27] O. Koteluk, A. Wartecki, S. Mazurek, I. Kołodziejczak, and A. Mackiewicz, "How Do Machines Learn? Artificial Intelligence as a New Era in Medicine," J. Pers. Med., vol. 11, no. 1, p. 32, Jan. 2021.

[28] A. Esteva et al., "A guide to deep learning in healthcare," Nat. Med., vol. 25, no. 1, Jan. 2019.

[29] P. Philipp, N. Merkle, K. Gand, and C. Gißke, "Continuous support for rehabilitation using machine learning," It - Inf. Technol., vol. 61, no. 5-6, 2019.

[30] O. Gottesman et al., "Guidelines for reinforcement learning in healthcare," Nat. Med., vol. 25, no. 1, pp. 16-18, Jan. 2019, doi: 10.1038/s41591-018-0310-5.

[31] M. J. Grant and A. Booth, "A typology of reviews: an analysis of 14 review types and associated methodologies: A typology of reviews, Maria J. Grant \& Andrew Booth," Health Inf. Libr. J., vol. 26, no. 2, pp. 91-108, Jun. 2009.

[32] G. Paré, M.-C. Trudel, M. Jaana, and S. Kitsiou, "Synthesizing information systems knowledge: A typology of literature reviews," Inf. Manage., vol. 52, no. 2, pp. 183-199, Mar. 2015.

[33] E. Aromataris, R. Fernandez, C. M. Godfrey, C. Holly, H. Khalil, and P. Tungpunkom, "Summarizing systematic reviews: methodological development, conduct and reporting of an umbrella review approach," Int. J. Evid. Based Healthc., vol. 13, no. 3, Sep. 2015.

[34] D. Moher, A. Liberati, J. Tetzlaff, D. G. Altman, and PRISMA Group, "Preferred Reporting Items for Systematic Reviews and Meta-Analyses: The PRISMA Statement," Ann. Intern. Med., vol. 151, no. 4, 2009.

[35] J. K. Kueper, A. L. Terry, M. Zwarenstein, and D. J. Lizotte, "Artificial Intelligence and Primary Care Research: A Scoping Review," Ann. Fam. Med., vol. 18, no. 3, pp. 250-258, May 2020.

[36] A. K. Triantafyllidis and A. Tsanas, "Applications of Machine Learning in Real-Life Digital Health
Interventions: Review of the Literature," J. Med. Internet Res., vol. 21, no. 4, p. e12286, Apr. 2019.

[37] B. Shickel, P. J. Tighe, A. Bihorac, and P. Rashidi, "Deep EHR: A Survey of Recent Advances in Deep Learning Techniques for Electronic Health Record (EHR) Analysis," IEEE J. Biomed. Health Inform., vol. 22, no. 5, pp. 1589-1604, 2018.

[38] T. G. Erdogan and A. Tarhan, "Systematic Mapping of Process Mining Studies in Healthcare," IEEE Access, vol. 6, pp. 24543-24567, 2018.

[39] E. Batista and A. Solanas, "Process mining in healthcare: a systematic review," in 2018 9th International Conference on Information, Intelligence, Systems and Applications (IISA), 2018, pp. 1-6.

[40] C. Xiao, E. Choi, and J. Sun, "Opportunities and challenges in developing deep learning models using electronic health records data: a systematic review," J. Am. Med. Inform. Assoc., vol. 25, no. 10, Oct. 2018.

[41] M. S. Islam, M. M. Hasan, X. Wang, H. D. Germack, and M. Noor-E-Alam, "A Systematic Review on Healthcare Analytics: Application and Theoretical Perspective of Data Mining," Healthcare, vol. 6, no. 2, Art. no. 2, Jun. 2018, doi: 10.3390/healthcare6020054.

[42] E. Choi, M. T. Bahadori, E. Searles, C. Coffey, and J. Sun, "Multi-layer Representation Learning for Medical Concepts," ArXiv160205568 Cs, Feb. 2016.

[43] Z. Zhu, C. Yin, B. Qian, Y. Cheng, J. Wei, and F. Wang, "Measuring Patient Similarities via a Deep Architecture with Medical Concept Embedding," in 2016 IEEE 16th International Conference on Data Mining (ICDM), Barcelona, Spain, Dec. 2016.

[44] E. Choi, A. Schuetz, W. F. Stewart, and J. Sun, "Medical Concept Representation Learning from Electronic Health Records and its Application on Heart Failure Prediction," ArXiv160203686 Cs, Jun. 2017, Accessed: Jun. 15, 2021.

[45] T. Ching et al., "Opportunities and obstacles for deep learning in biology and medicine," J. R. Soc. Interface, vol. 15 , no. 141, p. 20170387, Apr. 2018.

[46] R. Miotto, F. Wang, S. Wang, X. Jiang, and J. T. Dudley, "Deep learning for healthcare: review, opportunities and challenges," Brief. Bioinform., vol. 19, no. 6, pp. 1236-1246, Nov. 2018.

[47] H. K. Bharadwaj et al., "A Review on the Role of Machine Learning in Enabling IoT Based Healthcare Applications," IEEE Access, vol. 9, 2021.

[48] J. Enticott, A. Johnson, and H. Teede, "Learning health systems using data to drive healthcare improvement and impact: a systematic review," BMC Health Serv. Res., vol. 21, no. 1, p. 200, Mar. 2021.

[49] O. Koteluk, A. Wartecki, S. Mazurek, I. Kołodziejczak, and A. Mackiewicz, "How Do Machines Learn? Artificial Intelligence as a New Era in Medicine," J. Pers. Med., vol. 11, no. 1, Art. no. 1, Jan. 2021.

[50] G. Schryen et al., "Literature Reviews in IS Research: What Can Be Learnt from the Past and Other Fields?," Commun. Assoc. Inf. Syst., vol. 41, no. 1, Dec. 2017.

[51] L. Boecking and P. Philipp, "Recommeding Safe Actions by Learning from Sub-optimal Demonstrations," presented at the HealthRecSys, 2020. 